\title{
Monitoring and Analysis of Physical Exercise Effects Based on Multisensor Information Fusion
}

\author{
Xinliang Zhou $\mathbb{D}^{1}$ and Shantian Wen $\mathbb{D}^{2}$ \\ ${ }^{1}$ School of Physical Education, Xihua University, Chengdu, Sichuan 610039, China \\ ${ }^{2}$ School of Physical Education, Huzhou University, Huzhou, Zhejiang 313000, China \\ Correspondence should be addressed to Shantian Wen; wst@zjhu.edu.cn
}

Received 2 November 2021; Revised 10 December 2021; Accepted 15 December 2021; Published 10 January 2022

Academic Editor: Gengxin Sun

Copyright (c) 2022 Xinliang Zhou and Shantian Wen. This is an open access article distributed under the Creative Commons Attribution License, which permits unrestricted use, distribution, and reproduction in any medium, provided the original work is properly cited.

\begin{abstract}
In this paper, multiple sensors are used to track human physiological parameters during physical exercise, and data information fusion technology is used to extract useful information for monitoring and analyzing the effects of physical exercise. This paper explores the interaction and developmental dynamics of multisensor information fusion technology and physical exercise data monitoring based on the interrelationship and interpenetration between the two. The design ideas and principles that should be followed for the software designed in this study are discussed from the perspective of the portable design of measurement instruments and the perspective of multisensor information fusion, and then, the overall architecture and each functional module are studied to propose a scientific and reasonable design model. The general methodological model to be followed for the development of this resource is designed, and the basic development process of the model is explained and discussed, especially the requirement analysis and structural design, and how to build the development environment are explained in detail; secondly, based on the course unit development process in this model, we clarify the limitations of the system through meticulous analysis of the measurement results, which provides a solid foundation for the next step of system optimization. Finally, with a focus on future development, we elaborate on the potential possible role and development trend of multisensor information fusion in the future period. In this paper, we propose to apply the multisensor data fusion algorithm to the monitoring, analysis, and evaluation of the effect of physical exercise, by collecting multiple human physiological parameters during physical exercise through multiple sensors and performing data fusion processing on the collected physiological parameters to finally evaluate the effect of physical exercise.
\end{abstract}

\section{Introduction}

The method of tracking single sensor measurement data can no longer meet the growing requirements, and people have begun to explore the use of multiple sensor measurement data combined to maximize the extraction of useful information contained in the measurement data to achieve the tracking of moving targets [1]. When using measurement data from multiple sensors for target tracking, the measurement data provided by each sensor may have different characteristics, such as the sampling time, sampling location, data expression form, sampling frequency, and confidence level of each sensor to obtain the data, and the position of each sensor in the data fusion process is also different [2].
How to effectively fuse the measurement data from multiple sensors to obtain tracking performance that cannot be achieved by a single sensor has become the focus of research and attention in multisensor data fusion technology in the field of target tracking. Due to the rich information provided by many sensor data in the system that needs to be extracted and utilized, to better process the sensor data, combining intelligent theory with information fusion technology is an inevitable trend in the development of sensor information fusion technology. The development of multisensor information fusion technology has a wide range of applications in areas such as intelligent transportation, robotics, space navigation, and even military aspects. Nowadays, information fusion is increasingly used in life, and scholars around 
the world pay high attention to the development of multisensor information fusion technology. In data monitoring, it has also changed from a traditional single sensor to a multisensor composite [3].

Multisource information fusion technology, through the collection of multiple information sensors for multiple confirmations of different sensor systems, can be more conducive to improving the reliability of the sensor system and the ability to detect; conducive to improving the quality and credibility of the system's detection data and information; and conducive to improving the system's ability to perceive idiosyncratic data and data logical reasoning, accelerating the accuracy and response speed of the system, and improving the system's detection reliability; according to the data information provided by sensors of different information sources, through data fusion, it can improve the system's information decision-making and analysis capabilities, shorten the system's information response delay time, reduce the information ambiguity, and help to improve the system's information detection and processing performance [4]. Commonly used multisensor data fusion methods are the weighted average method, Kalman filter method, Bayesian estimation method, evidence inference method, fuzzy logic inference method, and artificial neural network method. Neural networks have strong nonlinear processing capability and meet the needs and technical requirements of multisensor data fusion. In the process of processing multisensor data fusion, the neural network model is used, and the learning algorithm inside learns the training samples, and eventually, through continuous learning, the neural network model is obtained, and then, the network model is used to obtain the signal processing capability, test the test samples, test the performance of the fusion model, and realize the fusion processing of multisensor data [5]. Among the many methods of data fusion, artificial neural networks have unique advantages in terms of the operating environment, information category, and applicability and a wide range of data fusion. Therefore, the research of applying neural networks for multisensor data fusion has received attention from various aspects of the world.

At this stage, in most Chinese young people, fast-paced daily life, work, and increased psychological pressure result in most Chinese young people's bodies in a state of subhealth and the emergence of some common chronic diseases; if this continues, without strengthening physical exercise and effective prevention, the physical condition of young people will become worse and worse, not conducive to the development of society. According to statistics, the number of deaths due to chronic diseases is increasing year by year, and they come rapidly, which for most of the Chinese aged people will cause huge socioeconomic and psychological pressure to their families in the light and even endanger their own lives in the heavy cases. Therefore, for the younger generation, it becomes especially important to exercise scientifically and effectively in fastpaced daily life [6].

A sports monitoring system based on multisensor information fusion is constructed in this project, which combines with IoT technology to achieve the monitoring of body temperature, blood pressure, EMG, and pulse during human exercise. The adaptive dynamic programming (ADP) data theoretical algorithm is applied in this human physical exercise monitoring system to fuse the physiological parameters obtained from the multisensors in the sensing layer of the system to realize the monitoring and judgment of the user's physical exercise condition.

\section{Current Status of Research}

With the rapid development of science and technology, there have been great advances in electronic technology, wireless communication technology, and biosensor monitoring. Health monitoring devices are developing towards integration, intelligence, multifunctionality, and portability [7]. The new health monitoring equipment has more comprehensive functions and monitors a wider range of human physiological parameters, and the structure and size of the whole equipment are more miniaturized. The core idea of the Bayesian filtering algorithm is to calculate the corresponding probability value according to the input data of the known sensor. According to the uncertainty of sensor measurement, it is expressed as conditional probability. Each sensor is estimated by Bayesian filtering, and finally, the calculated probability is calculated. The distribution combines a joint posterior probability distribution function. At this stage, the existing portable intelligent monitoring devices are mainly intelligent bracelets and intelligent clothing. A smart clothing company in Shenzhen, China, has developed a new type of smart clothing. This kind of clothing uses a self-developed flexible sensor, which has many different functions and soft models and can have certain deformation and other advantages [8]. It also has bioelectricity, pressure, and other sensor functions and can transmit bioelectric signals, pressure values, and respiratory frequency signals generated by human activities and obtain resting ECG/heart rate data, body posture parameters, and respiratory rate parameters. It can view the data via a mobile app and what measures should be taken for the current situation.

A smart company has developed a range of smart exercise clothing, with different kinds of clothing having different functions. Some garments can keep the body at a constant temperature, while others can make working out safer and reasonably efficient [9]. It also connects to your phone via Bluetooth and gives a more reasonable fitness plan based on data analysis. At present, smart monitoring equipment still has some shortcomings that need to be resolved, such as relatively high cost, short battery life, poor adaptability, and relatively simple functions and appearance structures [10]. Both can be made into the human body daily clothes to wear, can also be made into jewelry, and can also be made into smart helmets, smart glasses, and many other types. At present, manufacturers domestically and internationally have been using a variety of advanced technology to develop more advanced wearable clothing to meet the requirements of people's use.

The process of information processing is a core part of multisensor physical exercise monitoring through actual data reception, data processing with comparative analysis with existing knowledge and experience, and rational output results based on all acquired information [11]. Currently, 
there are algorithms for information fusion such as fuzzy algorithms, neural networks, mutual correlation algorithms, and specific relational equations. The neural network algorithm is considered one of these information fusion algorithms with better accuracy in multisensor fire detection and is widely used by people in various fields. Compared with the traditional single sensor, these processing methods have obvious essential differences in the processing methods of the detection signal. It is now more common to classify according to the processing level and level of fusion, which is divided into three levels: information layer fusion, feature layer fusion, and decision-making layer fusion. Neural networks are a class of physiologically based intelligent bionic models that simulate the working principles of the human brain to deal with problems, consisting of a large number of processing units in a nonlinear adaptive dynamic system with good self-adaptability, self-organization, and strong learning, associative fault tolerance, and anti-interference capabilities [12]. The advantages of this technology are reflected in the fields of pattern classification recognition and nonlinear curve fitting. The neural network is a research hotspot in the field of artificial intelligence development. When the neural network is applied to multisensor data fusion, the first step is to select a suitable neural network model, and the selection criteria are the requirements of the fusion system and the characteristics of the sensors, including the topology of the network, neuron characteristics, and learning rules [13]. It is also necessary to establish connections between the input and sensor information and between the output and the system decision and then determine the assignment of weights based on the acquired sensor information and the corresponding system decision information to complete the training of the network [14]. When the sensor is accurate, we can correctly determine the health of the human body. If the accuracy of the sensor is insufficient, it will greatly affect the recognition of the human health and may even cause undesirable consequences. Therefore, special attention should be paid to the accuracy and stability of the sensor. After training the neural network which can then participate in the actual fusion process, the data obtained from the sensors are first processed appropriately by the process and then used as the input to the neural network, which is processed by the neural network and finally sent to the processing to interpret it as the system-specific decision behavior; the BP neural network is currently the most widely used network. Many researchers are currently working on neural networks in the field of multisensor data monitoring with satisfactory results.

\section{Design of a Physical Exercise Monitoring System with Multisensor Information Fusion}

3.1. Design of a Multisensor Physical Exercise Monitoring System. In the multisensor information system, the multisensor information has a variety of manifestations, huge information capacity, complex correlations between various types of multisensor information, and high requirements for the timeliness of information acquisition and processing, which requires an effective method to collect and process multisensor information acquired by multilevel sensors in the multisensor system, and through the coordination and performance complementarity among multisensors, to quickly and effectively to derive a comprehensive and correct understanding of the surveillance (detection target) object [15]. The research design of this paper is a monitoring system of human physiological parameters for physical exercise, which is mainly used for real-time monitoring of physical exercise in the daily life of families and individuals and real-time feedback of the physiological parameters of human movement. After an in-depth understanding and study of the functions to be achieved and the technical theoretical knowledge required, the overall structure and functions of the system are systematically designed and studied. The system is divided into three parts: information acquisition, information judgment, and information display as shown in Figure 1.

The system establishes a hardware architecture based on a Wi-Fi network, uses an embedded Wi-Fi microcontroller as the processing core of the system, and uses Java programming language to design and develop a software application platform for the remote health monitoring systems. The system detects four basic physiological parameters of the human body: pulse, heart rate, blood pressure, and body temperature from multiple information sources, and combines the concept of data fusion to give a judgment of whether the human body is in a healthy state. At the same time, the system also designs the functions of remote realtime monitoring of body indicators, intelligent heart rate monitoring and first aid, and automatic storage of health data according to actual needs [16]. Excessive sweating and insufficient rehydration can also lead to dehydration and affect health. In the case of heavy sweating, conditions such as thirst, oliguria, fatigue, muscle cramps, and heat cramps may occur. In severe cases, symptoms of heat stroke may occur and may even be life-threatening. Data on basic human physiological parameters of the user (pulse, EMG, blood pressure, and body temperature) are collected through wearable devices that are in contact with the body. After obtaining the data, they are sent to the processor and simply classified. The various physiological indicators of the user collected by the human body indicator sensor collection terminal are processed by the Wi-Fi module and then uploaded to the server in real time through the Wi-Fi wireless network; i.e., in this system, the Wi-Fi module acts as both the communication module and the only processor in the system. To address the binding and identification of data information, a unique ID is assigned to each registered user on the server side, and users can view their information related to this vital sign signal through the smart mobile terminal-based app. If the physiological indicators are out of the preset range, the system will display the corresponding alarm information and the alarm will be automatically alerted. At this time, the user can choose to store the abnormal physiological indicator information in the database for future analysis.

$$
\frac{x_{m}-\sqrt{x^{2}+m^{2}}}{x_{m}} \leq e .
$$




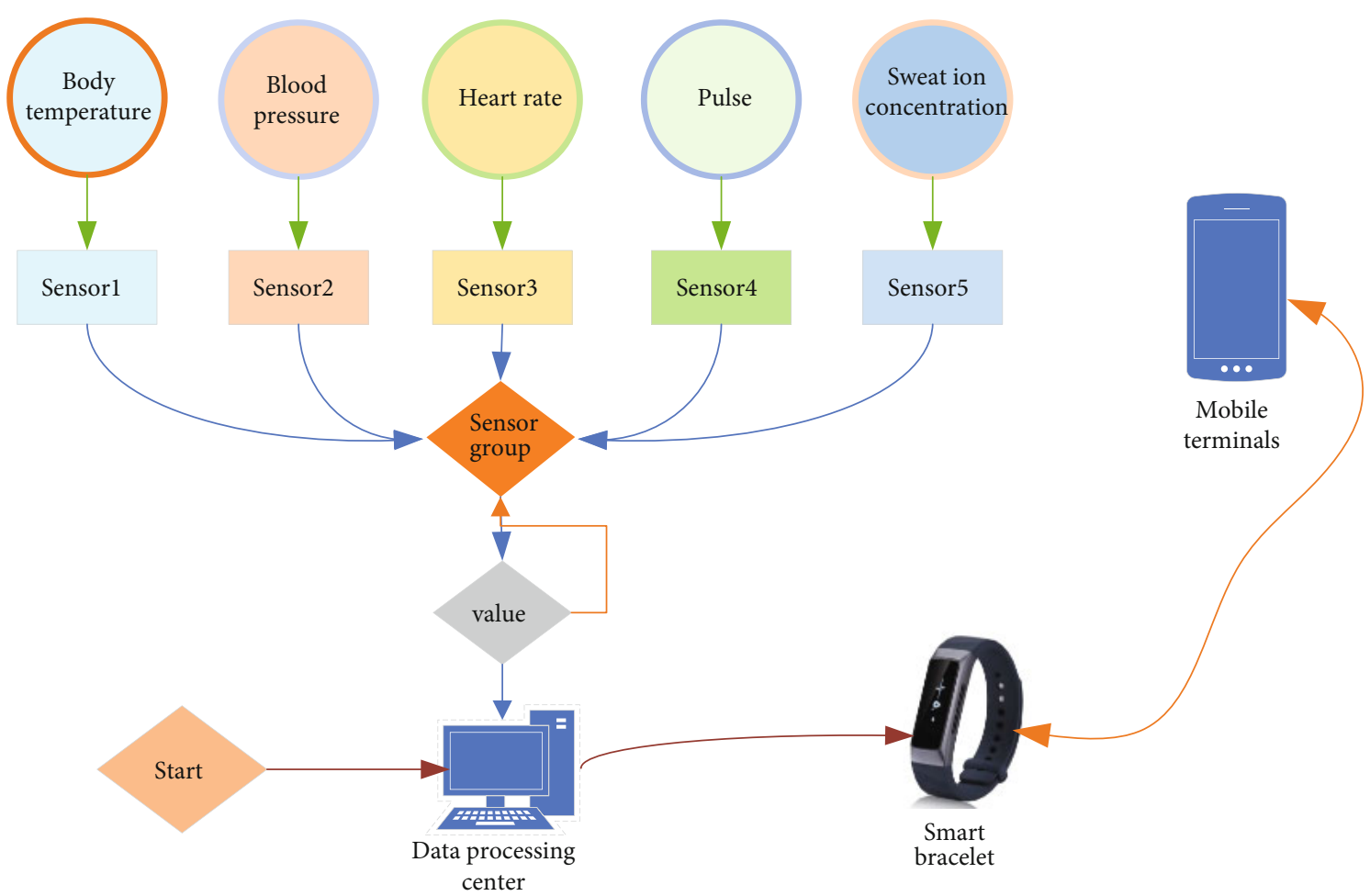

FIGURE 1: Structure flow of the physical exercise monitoring system.

This paper discusses a multilevel multisensor information acquisition system, which mainly completes the collection and acquisition of detection data from radar sensors, photoelectric sensors, and ground sensors, and performs the necessary data preprocessing. The multisensor information acquisition system is the basis for application-specific surveillance information systems for fusion processing, information monitoring, and information dissemination, providing them with raw surveillance data information. The information quality provided by multilevel multisensor directly affects the quality and performance of multisensor information fusion output. Multisensor systems in surveillance information systems are often covered according to partitions, which form multisensor information acquisition systems. Multilevel multisensor system partitioning is shown in Figure 2.

A fiber optic radiation temperature sensor can be used to measure the body temperature signal and output the $\mathrm{AD}$ value of the measured body temperature. A fiber optic absorption probe has the obvious advantages of small size, high accuracy, small interaction with the electromagnetic field, and high sensitivity, but the measurement range is relatively narrow. Because the measurement of a specific part of the human body can be obtained from this part of the human body temperature, the measurement range has small impact on the accurate measurement of body temperature, so you can choose the fiber optic radiation temperature sensor to measure body temperature. Since the fiber optic diameter is small and malleable, multiple probes can be used to measure body temperature in different parts of the body, constituting a multisource temperature measurement system. The body temperature acquisition module is selected from Huaqiang Electronics manufacturer's V1.1 body temperature acquisition sensor [17]. After comparing with other sensors in terms of accuracy, stability, size, sensitivity, and other aspects, this temperature acquisition module has certain advantages. The operating characteristic of this temperature acquisition module is that the voltage decreases as the temperature increases. The typical feature of this acquisition module is that it is sensitive to temperature and can be accurate to two decimal places, which is especially suitable for small changes in the human body temperature range under the influence of various conditions, and the temperature range is exactly in line with the human body temperature range. This section conducts an overall test of the physical exercise system and compares the stability, reliability of the system, and the accuracy of the physiological data collected by the system. Using the nonlinear fitting ability of neural network, through the nature of the data correlation between sensors in the multisensor system, the accurate prediction value is used to diagnose whether the sensor is faulty and the fault location and to repair the data of the faulty sensor. The linear range of voltage change is 30-4 degrees/voltage $2.127-1.193 \mathrm{~V}$, and the temperature characteristic curve is shown in Figure 3.

Pulse check is an auxiliary test used to check the normal functional status of the circulatory system. The human circulatory system is responsible for transporting various nutrients and waste products in the body, and the blood acts as a carrier in a never-ending work. During the cardiac cycle, periodic pulses of dilation and vascular return occur because of alternating ventricular systole and diastole. The 


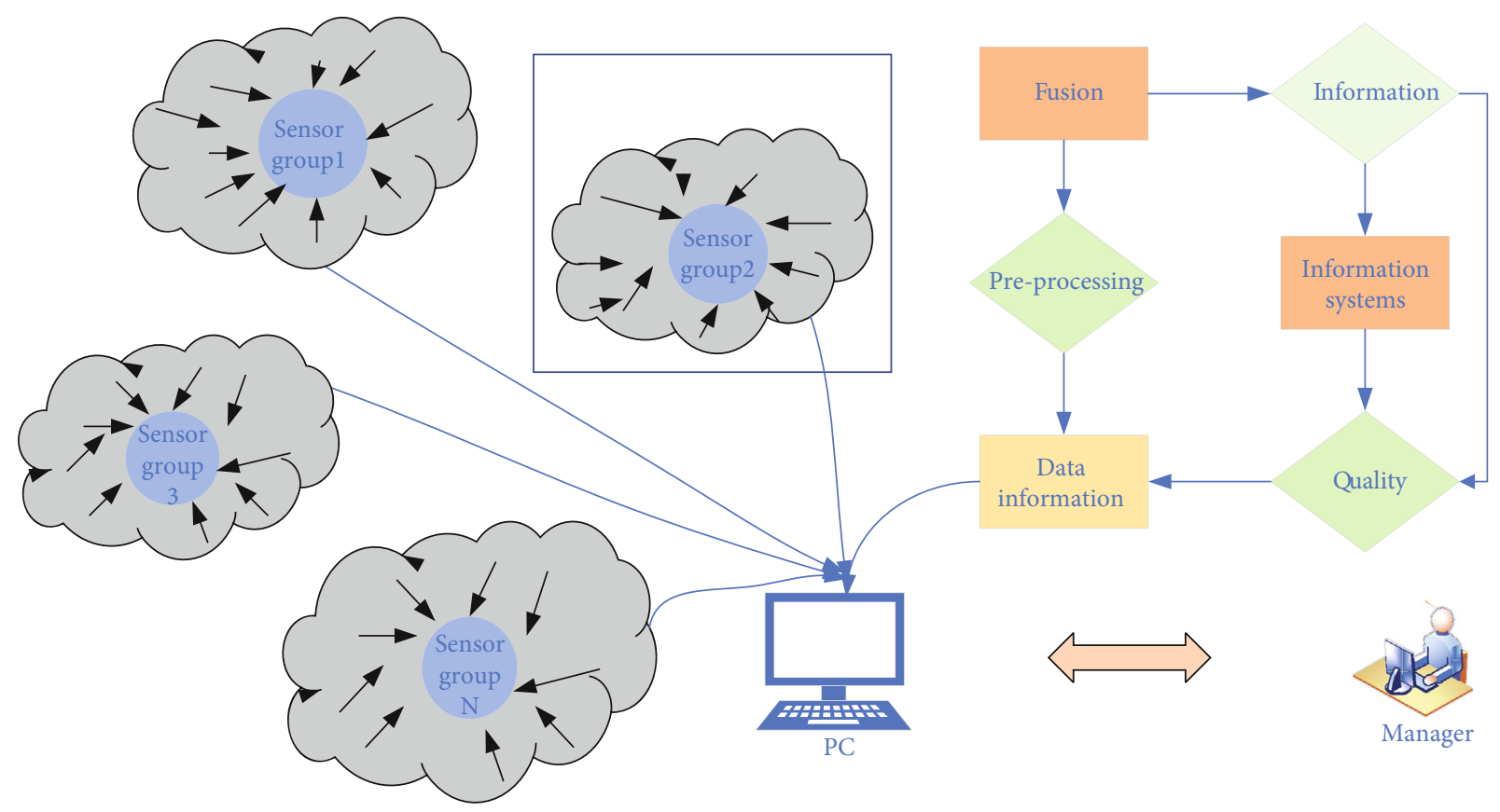

FIGURE 2: Schematic diagram of the partitioned coverage of a multisensor system.



FIGURE 3: Temperature characteristic curve of physical exercise.

pulse rate changes significantly when the body is in poor condition, especially when critically ill. Changes in the pulse rate are also used by doctors as an important indicator in the diagnosis of a condition. A pulse check includes the examination of the rate, rhythm, strength, and waveform of the pulse. In this article, the pulse test uses a pulse sensor to detect the pulse and output a digital signal value. The probe of the sensor is applied to the skin surface where the arterial beat is strong and a certain pressure is applied.
The micropressure material in the probe picks up the pressure signal of the pulse beat, and an electrical signal change is generated, which is first amplified and then processed by the adjustment circuit to obtain the complete waveform of the pulse beat, which further gives a set of pulse signals synchronized to the pulse beat. Piezoelectric and piezoresistive pulse sensors are mainly used in clinical applications for the pathological analysis of cardiovascular diseases such as atherosclerosis. 


$$
x=\frac{X-\max (X)}{\max \left(X^{2}\right)+\min (X)} .
$$

3.2. Multisensor Data Fusion Processing. Data preprocessing is an important prerequisite for multisensor data fusion. Due to the relatively large variation in the range of variation of various physiological parameters during exercise, unprocessed raw data from sensors often have missing data, misleading data, data not in line with common sense, and other problems, resulting in data analysis being not easy. To analyze the data reasonably, we usually must perform preprocessing operations on the raw data to normalize the raw sensor data. The specific applications of multisensor information fusion technology in the military field mainly include marine surveillance and air-to-air and surface-toair defense systems. The marine monitoring system mainly detects, tracks, and identifies military targets. Air-to-air and surface-to-air defense systems mainly detect, track, and identify enemy aircraft, missiles, and air defense weapons. After normalization of the original data, the data will be stored in plain text form after preprocessing, which is convenient for subsequent programming. The multisensor information is collected through the multisensor data collection interface, and the collected multisensor information is distributed to the upper-level monitoring center using the multisensor information distribution service, thus completing a system with two or more levels of multisensor information collection. The types of multisensor information in the system mainly include radar information, optoelectronic information, ground sensor information, and navigation and positioning information, and the multisensor information acquisition system acquires various types of multisensor information in real time by adapting different types of sensor acquisition interfaces, as shown in Figure 4.

For the "surveillance information system + RS + GPS + GIS," the core problem of research and solution is that it should make full use of the complementary or redundant multisensor in the time and space domain to carry out the relevant coordination, data processing, and synthesis of multisource information. There is no clear boundary between various sensor information sources, multisensor systems, integrated situational systems, information fusion, and auxiliary decision support systems in the whole surveillance information system, and there is information coupling and feedback between various systems [18]. Therefore, the multilevel multisensor information acquisition system can obtain two or more levels of multidimensional surveillance target information. At present, in this field, multisensor information fusion technology is mainly used to fuse the information collected by each sensor in the smart car, to determine the road position, the roadblock ahead, the speed limit, and other information of the smart car, and then, according to the information in the system, the corresponding rules can realize autonomous driving of smart cars.

$$
y\left(x^{i}\right)=v_{0}^{\prime}-\sum_{i=1}^{n} \kappa_{i}\left(x^{i}\right)^{2} .
$$

In the target environment, a single sensor can only capture a certain type of information of the target environment and cannot form a complete description of the target environment. And the information fusion mode of a single sensor is also relatively low level, simply imitating the process of processing primary information in the human brain, and cannot extract the effective information needed for the target environment. With heterogeneous multiple sensors, the type of information collected for the target environment is different due to the different sensor types. Therefore, this rich source of information and information collection is followed by a multisensor information fusion technique that can more fully extract the valid information in the target environment, resulting in a more comprehensive and accurate description of the target environment. Each multisensor information fusion mode has its advantages and disadvantages. The multisensor data fusion algorithm is applied to the monitoring and analysis of the effect of physical exercise, a variety of human exercise physiological parameters are collected through the multisensor data network, and the collected human physiological parameters are subjected to data fusion calculation, and finally, the physical exercise effect is obtained. For example, the data-level fusion mode has the advantage of a more comprehensive amount of original information, which is what feature-level fusion lacks, and the less computational information processed by feature-level fusion is also an advantage that data-level fusion does not have. Therefore, in the process of fusing specific target environment information in specific application areas, the advantages and disadvantages of each mode can be combined and different multisensor information fusion algorithms can be used flexibly at different levels, thus improving the ability to accurately describe the target environment.

Tracking of moving objects requires filtering out noise in noise-containing data and minimizing or even eliminating the effect of noise on the tracking system to obtain an optimal estimate of the target's motion state. The Kalman filtering algorithm is the most classical and commonly used target tracking filtering algorithm, if the tracking system satisfies the linear and Gaussian white noise condition, using the minimum variance criterion to estimate the tracking object motion state recursive processing method. The Kalman filter algorithm not only calculates the optimal estimate of the tracking object's motion state at a given moment but also calculates the error covariance matrix. A theoretical framework for optimal tracker design is provided for tracking problems with known target system equations and observation equations. The method of tracking the measurement data of a single sensor can no longer meet the increasing requirements. People have begun to explore the comprehensive utilization of the measurement data of multiple sensors to maximize the extraction of useful information contained in the measurement data to achieve tracking of moving targets. However, practical target tracking systems are often nonlinear, and the traditional Kalman filtering algorithm cannot handle nonlinear tracking problems well. The nonlinear filtering of target tracking, i.e., the estimation of the posterior probability distribution of the 


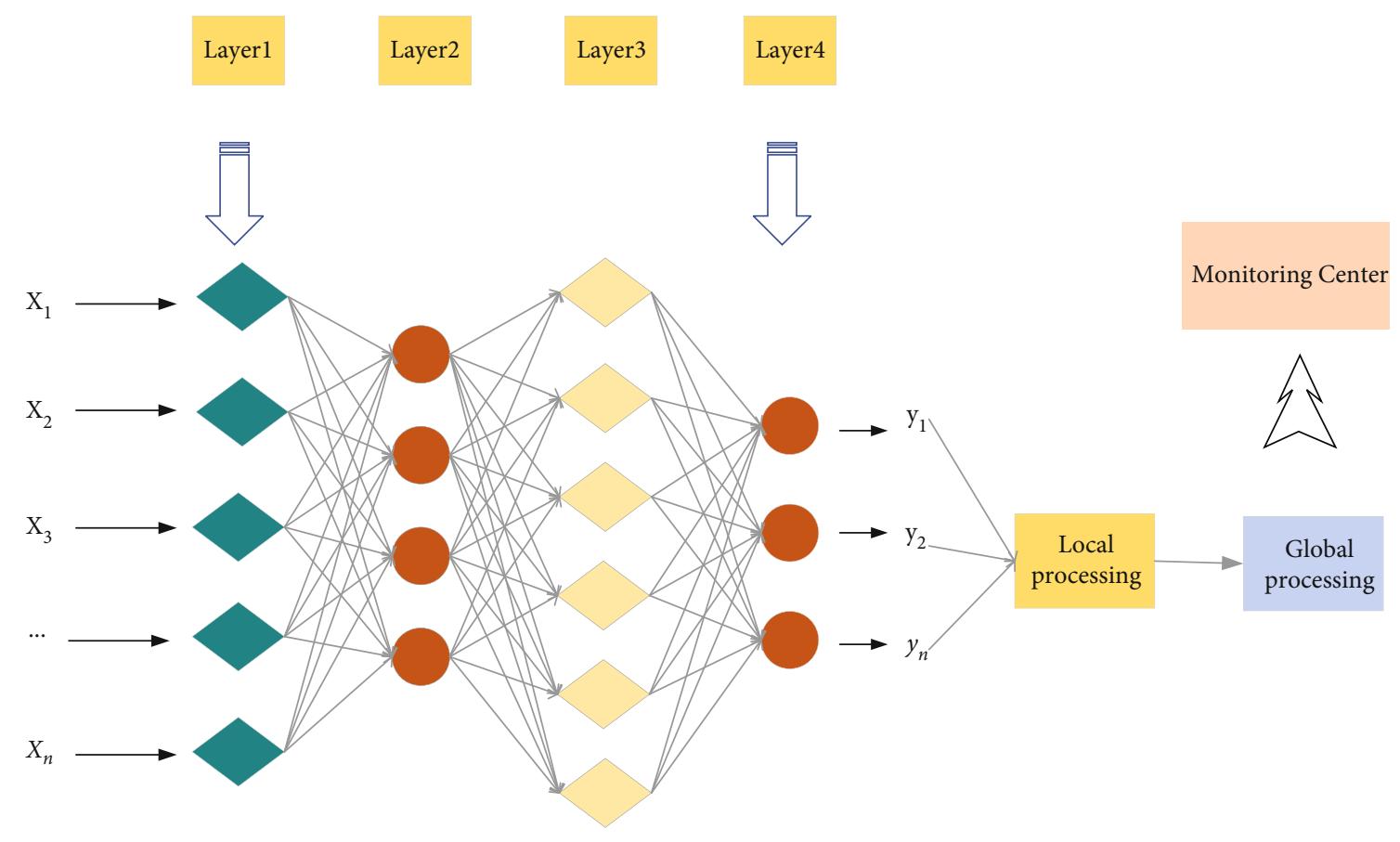

FIGURE 4: Network structure model of multilevel multisensor information acquisition system.

moment state under the observation sequence condition, is expressed by the formula, where the optimal method to deal with the nonlinear tracking filtering problem is to obtain the posterior probability distribution of the target motion state; however, obtaining the exact posterior probability distribution requires a large amount of data, which cannot be realized in the practical application system, and some suboptimal approximation methods which have been proposed for these commonly used nonlinear target tracking filtering algorithms are discussed below.

$$
c_{k}=\int p\left(z_{k+1} \mid x_{k}\right) p\left(x_{1: k+2} \mid z_{k}\right) d, \quad k=1,2, \cdots .
$$

Many classical filtering and fusion ideas originate from Bayesian filtering methods, such as particle filtering, Kalman filtering, and other algorithms which are specific implementations of Bayesian filtering algorithms. Multisource information fusion technology, by collecting multiple information sensors to confirm different sensor systems multiple times, can be more conducive to improving the reliability and detection capabilities of the sensor system; it is conducive to improving the quality and credibility of the system detection data and information degree and is conducive to improving the system's ability to perceive specific data and data logical reasoning, speed up the accuracy and response speed of the system, and improve the detection reliability of the system. The core idea of the Bayesian filtering algorithm is to calculate the corresponding probability values based on known sensor input data, represent the uncertainty of sensor measurements in terms of conditional probabilities, make Bayesian filtering estimates for each sensor, and finally synthesize the calculated probability distributions into a joint posterior probability distribution function.
The environmental features detected by the prior model are characterized by computing the sensor fusion weights derived from the distribution function. Bayesian filtering is characterized by the fact that all estimates are based on probability distributions rather than specific values, and the goal of the Bayesian filtering algorithm is to combine the state probability distributions of the previous moment to estimate the current moment state quantities based on the control and observation model. Also, Bayesian filtering is a general term for a large class of methods, an abstract expression, and a concrete implementation of the Kalman filter fusion algorithm. The Kalman filter algorithm is based on the prerequisite that an accurate mathematical model is known. At this stage, the fast-paced daily life, work, and increased psychological pressures of most Chinese young people have caused most Chinese young people's bodies to be in a subhealthy state and have some common chronic diseases. If this goes on, they will not strengthen physical education. With exercise and effective prevention, the physical condition of young people will get worse and worse, which is not conducive to the development of society. However, building accurate mathematical models for nonlinear target tracking systems is difficult. In practical target tracking systems, it happens that the target state model and observation model do not match the actual system, which is a case of partial prior knowledge uncertainty of the target model from the estimation theory point of view. To solve the problem of mismatch between the mathematical model established in the target tracking system and the actual system, the adaptive Kalman filtering algorithm is generated. Adaptive Kalman filtering uses the information carried by the newly acquired measurement data to change the Kalman gain matrix and the system noise of the target tracking problem online to seek an approximation of the optimal filtering 
and to solve the mismatch between the system modeling and the actual motion of the target.

$$
\begin{gathered}
\phi(k)=\left[\begin{array}{ccc}
T & 0 & \frac{T}{2} \\
1 & 0 & 0 \\
1 & T^{2} & 1
\end{array}\right], \\
f(x)=\operatorname{sgn}\left(\sum_{i=1}^{l} \alpha_{i}^{x} \Phi\left(x_{i}\right)-y_{i} b^{*}\right) .
\end{gathered}
$$

Multisensor is the basis of information fusion, multisensor information is the object of processing of information fusion, and integrated processing is the core of information fusion; multisensor information fusion is performed by combining the advantages of multiple single sensors, reasonably mining the use of the detected fire characteristic information and fusing the spatially and temporally complementary or redundant information according to certain criteria to obtain a unified conclusion about the observed object. Information fusion can be classified by its fusion structure, algorithms, techniques, etc. [19]. The neural network is a research hotspot in the field of artificial intelligence development. When the neural network is applied to multisensor data fusion, the appropriate neural network model must first be selected. The selection criteria are the requirements of the fusion system and the characteristics of the sensor, including the network, the topological structure, neuron characteristics, and learning rules etc. At the same time, it is also necessary to establish a connection between input and sensor information and between output and system decision-making and then determine the distribution of weights according to the acquired sensor information and corresponding system decision-making information to complete the training of the network. There are clear and fundamental differences in the way these processing methods treat the detection signal compared to traditional single sensors. Nowadays, it is more common to classify them by the level and hierarchy of processing of fusion, which is divided into three levels: information-level fusion, feature-level fusion, and decision-level fusion. The information fusion level system focuses on how to analyze and process the multisensor detection information at different processing stages. The higher the fusion level, the less detail is required for the original information, but the corresponding level of abstraction is also higher.

$$
K\left(X_{1}, X_{2}\right)=\left[\left(X_{1}^{T^{2}}, X_{2}\right)\right]^{\ell} .
$$

Time synchronization of different sensors in the multisensor fusion process is very important, but the basis of time synchronization is to ensure that each sensor uses the same clock source and the information to be collected in the same moment of data. Because the sensor clocks used generally have drift errors and each clock source has a different drift, initial moment timestamp alignment is required in the multisensor fusion process, but with a period of operation, the aligned timestamps will still be misaligned by drift. The timestamp synchronization method used in this thesis is to design a hardware pulse generator to unify the trigger pulses of all sensors, and each pulse trigger can correct and eliminate the clock source drift, as shown in Figure 5.

\section{Performance Results of a Multisensor Physical Exercise System}

The importance of body temperature to the human body is self-evident, and special attention should be paid to the monitoring of human body temperature when monitoring the health condition of the human body. When the system is running, check that the whole system is in normal connection, open the upper computer software interface, and supply power to the whole system. In the port setting interface of the upper computer LabVIEW, select the COM port and baud rate values to ensure that the Arduino is successfully connected to LabVIEW. The user enters information such as name, gender, age, mobile phone, landline, and notes in the information input interface, places the sensor on the appropriate acquisition site, and clicks start, and the system collects and reads the data and displays the acquired data and waveform graph in the data display interface. The data analysis interface will show the specific situation of human health. When detecting the body temperature signal, a fiber optic radiation temperature sensor can be used to measure the body temperature signal and output the $\mathrm{AD}$ value of the measured body temperature. The optical fiber absorption probe has obvious advantages such as small size, high accuracy, small interaction with electromagnetic fields, and high sensitivity, but the measurement range is relatively narrow.

By testing the overall operation of the system, the test obtained that the system can operate stably and continuously, indicating that the system is normal. After that, the stability and accuracy of the sensors are tested. The accuracy and stability of the sensor are extremely important for the whole health monitoring system. When the sensor measurement is accurate, we can correctly determine the health of the human body; if the accuracy of the sensor is not enough, it will greatly affect the identification of human health conditions and may even cause adverse consequences. Therefore, special attention should be paid to the accuracy and stability of the sensor. Body temperature usually refers to the internal temperature of the human body. In daily life, mercury thermometers are commonly used to measure the axillary body temperature, which is normally in the range of 36-37 degrees Celsius. Body temperature is susceptible to various internal and external factors, so body temperature can vary slightly within the normal range, usually by no more than 1 degree Celsius, and this change is not harmful to human health. Due to the relatively large variation range of various physiological parameters during exercise, the unprocessed raw data of the sensor often appears: data missing, disordered data, and data inconsistent with common sense, which makes data analysis difficult. To analyze the data reasonably, we usually need to preprocess the raw data to standardize the raw data of the sensor. For example, in a physiological state, the body temperature is at a low value in the morning and 


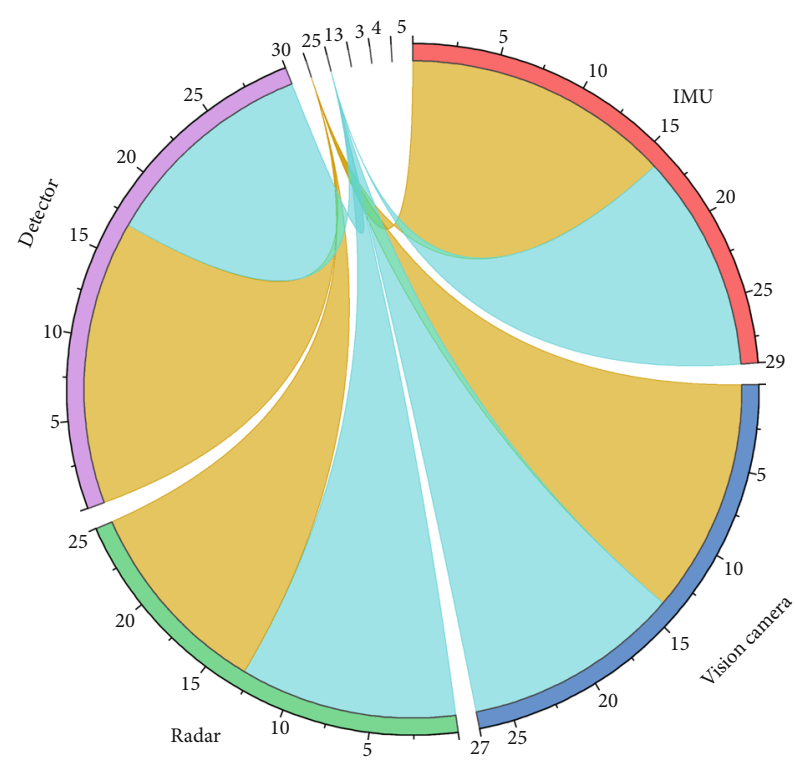

FIGURE 5: Multisensor time synchronization comparison schematic.

will rise slightly in the afternoon. Suddenly entering a hot environment or emotional stress can also cause a slight increase in body temperature. There are also slight differences in body temperature by age; for example, children have a slightly higher body temperature compared to adults, while older people have a slightly lower body temperature. The relative constancy of body temperature is one of the important basic conditions for maintaining normal life activities in the human body.

The information revealed by the human heart rate reflects the health of the human heart. The heart rate is the number of times the heart beats in a minute, and there are two different states. Quiet heart rate is the size of the human heart rate when in a quiet state, and the normal adult heart rate value range is generally 60-100 beats. The maximum heart rate of a person refers to the maximum number of times the heart beats in a minute. The heart rate of adults is generally between 60 and 100 times/min, most people are in the 60-80 times/min, and women generally have faster heart beat than men; for children, the heart rate may be higher generally in the 100 times/min or more; for the elderly due to the aging of the body organs, the heart rate will have a certain degree of reduction. It can be seen in heavy physical work for long periods and athletes. Athletes have a slower heart rate than the average adult, typically around 50 beats per minute. In the quiet state, there are some differences in the heart rates of adults and athletes, as shown in Table 1.

Most of the sweat is water, and this also contains minerals such as sodium, potassium, chloride, magnesium, calcium, and phosphorus. The Kalman filter algorithm can not only calculate the optimal estimation of the motion state of the tracking object at a certain moment but also calculate the error covariance matrix. It provides a theoretical framework for optimal tracker design for the tracking of known target system equations and observation equations. The most ions in sweat are sodium and chloride, with some
TABLe 1: Heart rate in different exercise states.

\begin{tabular}{lcccc}
\hline Status & Kids & Females & Male & The elderly \\
\hline Normal & 99 & 77 & 100 & 90 \\
Slow walking & 102 & 73 & 92 & 55 \\
Rush walking & 110 & 85 & 70 & 74 \\
Jogging & 96 & 94 & 81 & 57 \\
Running & 96 & 75 & 66 & 73 \\
Vigorous exercise & 100 & 66 & 80 & 50 \\
\hline
\end{tabular}

amount of potassium and calcium ions also present. People sweat a lot during some strenuous physical activity, and it is mainly sodium ions that are lost with sweat. The body relies on sodium and chloride ions to regulate fluids and body temperature, and these two ions are important to the body. Although sweating is a physiological regulation, heavy sweating and insufficient rehydration will also lead to dehydration and affect your health. A relatively large amount of sweating may produce conditions such as thirst, low urination, fatigue, muscle cramps, and heat cramps. In severe cases, symptoms of heatstroke may occur and may even be life-threatening [20]. When the body exercises vigorously, it also loses large amounts of mineral ions after losing large amounts of sweat. The $\mathrm{pH}$ balance in the body is then affected to some extent, the $\mathrm{pH}$ level changes and the body's sweat ratio is lower than normal. The importance of sweat for the human body cannot be overstated, and it also reflects the health of the body to some extent. The measurement of human health can also be done by collecting and monitoring the sweating of the body and the ion concentration of sweat. The ion concentration in human sweat also reflects some of the health information of the human body, as shown in Figure 6.

This section provides an overall test of the physical exercise system, comparing the stability and reliability of the system as well as the accuracy of the physiological data collected by the system. The nonlinear fitting capability of the neural network is used to diagnose whether a sensor is faulty and the fault location by the nature of data correlation between sensors in a multisensor system using accurate predictive values and repairing the data from the faulty sensor. The role of the BP neural network in the sensor fault diagnosis structure proposed in this chapter is to fuse the correlation data between individual sensors, and the fault diagnosis relies on the prediction value errors of these fusion models to determine the fault of the system and to recover the faulty sensor data. For the three typical forms of sensor faults detected by the simulation experiments, the data fusion sensor fault diagnosis model proposed in this paper can accurately detect the faults and remedy them effectively, which proves that such a sensor diagnosis model is effective. It ensures that the system can operate continuously and stably in actual use, the measured physiological data are accurate and reliable, and the human exercise physiological parameters obtained after multiconditional judgment are correct.

The average value of the athlete's reaction to action is $46.82 \mathrm{~ms}$. The IAAF's starting reaction limit is $100 \mathrm{~ms}$. The 


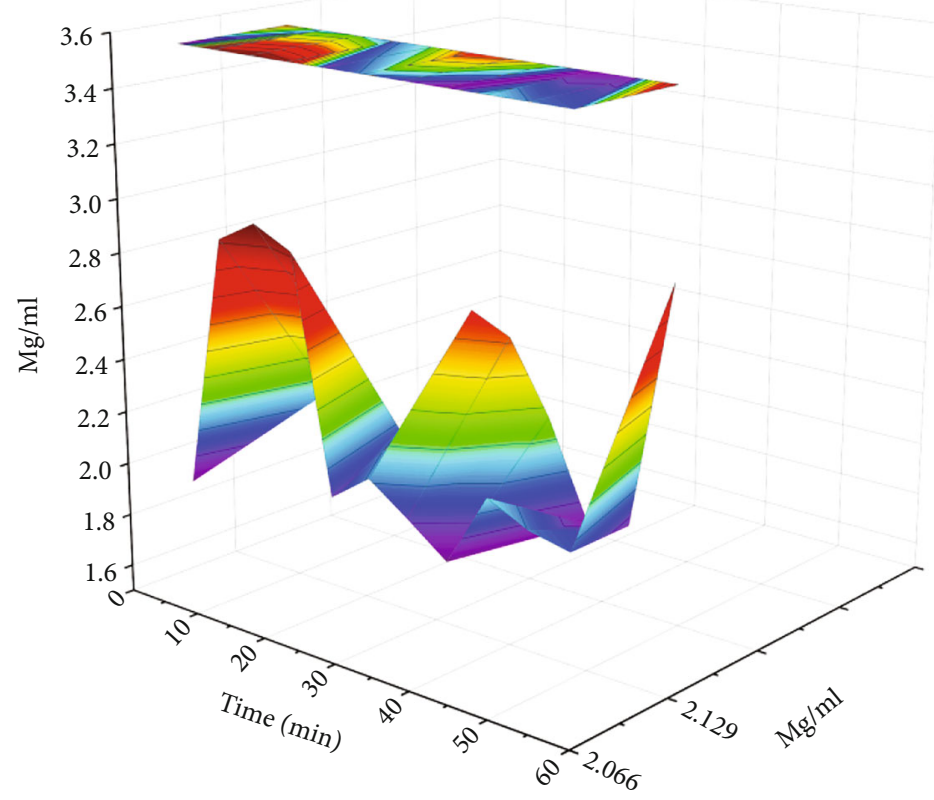

FIGURE 6: Sweat ion concentration in different exercise states.

average value of the athlete's reaction to action accounts for $46.82 \%$ of the starting reaction limit. The starting reaction limit includes the reaction to action, so the error of judging whether an athlete fouls a foul is also $46.82 \%$. Athlete's reaction to action has an important influence on whether the athlete's starting reaction exceeds the limit and fouls. When reacting to the action, the fastest is the 8th track in the men's $100 \mathrm{~m}$ final with a time of $15 \mathrm{~ms}$, and the slowest is the 4th track in the first group of the men's $200 \mathrm{M}$ preliminaries with a time of $143 \mathrm{~ms}$; the difference between the two is $128 \mathrm{~ms}$. This shows that under the current electronic starting monitoring system, if the actual reaction time of two athletes is the same, then the first reaction time is $128 \mathrm{~ms}$ faster than the latter's starting reaction time. Therefore, under the same conditions, the former has a starting reaction time compared to the latter. The possibility of overlimit fouls has increased significantly.

\section{Results of Multisensor Information Fusion Applications}

Multisensor information fusion has a wide range of applications, and its specific applications in the military and civilian domains are briefly described below. In the military field, certain geographic locations or military activity areas are restricted by various factors, making it impossible for military personnel to make accurate judgments about the target environment. The emergence of multisensor information fusion technology is a good solution to this dilemma. Applying multisensor information fusion technology to this field can achieve accurate detection, precise positioning, tracking, and identification of the target environment, thus providing military commanders with more reliable battlefield information for effective judgment of the next military operation. The specific applications of multisensor information fusion technology in the military field are mainly marine surveillance, air-to-air and ground-to-air defense systems, etc. The ocean surveillance system mainly detects, tracks, and identifies military targets. The air-to-air and ground-to-air defense system mainly detects, tracks, and identifies enemy aircraft, missiles, and air defense weapons. At present, the main applications of sensors include marine defense, antisubmarine warfare, detection of stealth aircraft, artillery, electric warning, tracking enemy military actions, and preventing biochemical attacks as shown in Figure 7.

Multisensor information fusion technology is mainly used to fuse and reason with the information collected from video sensors, sound sensors, etc. in the robot and then combine it with the robot's own implanted operating program to perform the target task to complete the prespecified work in the field of intelligent transportation. At present, in this field, multisensor information fusion technology is mainly used to fuse the information collected from each sensor in the smart car, to determine the road location, roadblocks ahead, speed limits, and other information of the smart card, and then realize the autonomous driving of the smart card according to the corresponding rules in the system [21]. Multisensor information fusion technology is mainly through the information collected by infrared sensors, microwave sensors, etc., to detect the location of the hidden weapons or drug situation fusion analysis and finally get the fusion results to determine the site conditions. At the same time in this field, multisensor information fusion technology can also be an effective fusion of the information collected from the face, fingerprints, voice, etc., to improve the identification and authentication of the suspect, greatly improving the efficiency of crime-solving.

In the past medical diagnosis, surgeons often used visual inspection, thermometers, or stethoscopes to aid in diagnosis. More advanced and effective medical sensing 


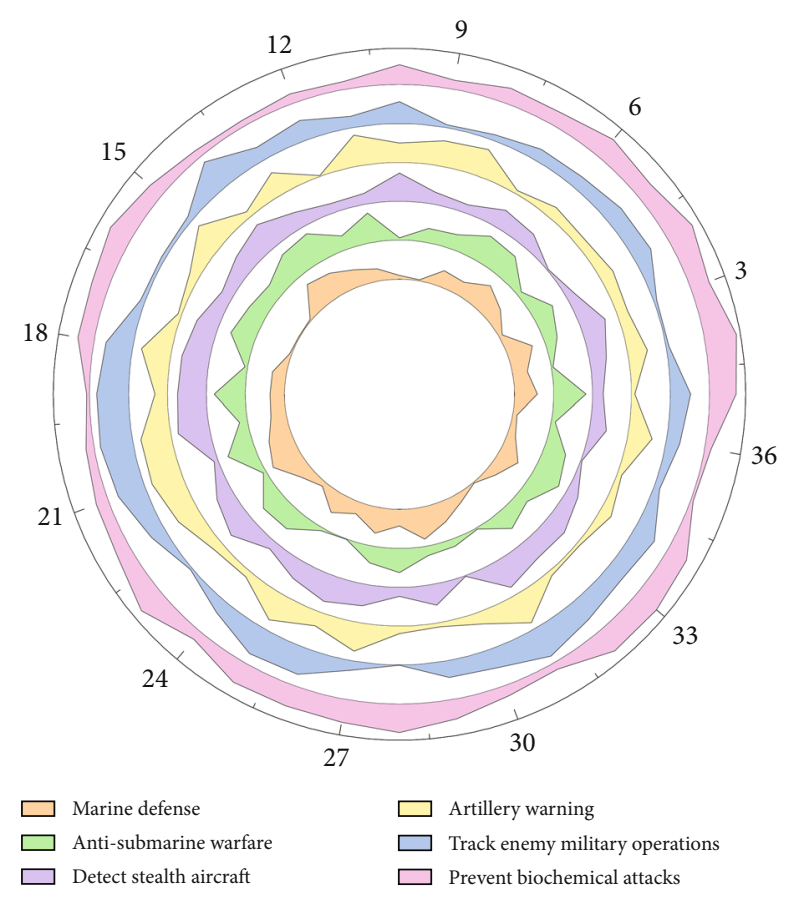

Figure 7: Application result.

technologies have now emerged, such as ultrasound imaging, localization, and identification of tumors. The use of information collected by these sensors and the effective fusion of this information has made modern medical diagnosis much more accurate and efficient.

\section{Conclusion}

Internet of Things (IoT) technology is rapidly booming and has become a newly developed norm in the process of modern people achieving information management in their daily lives. The integration of Internet data applied in wireless medical and health services is also increasing. Due to the high intensity of life and work pressure in modern society, people have less and less time to participate in physical exercise, and increased people are in a subhealthy or even unhealthy state. Therefore, how to carry out physical exercise scientifically and effectively is gaining increased attention. In this paper, we propose to apply the multisensor data fusion algorithm to monitor and analyze the effect of physical exercise and collect multiple human exercise physiological parameters through a multisensor data network and calculate the collected human physiological parameters by data fusion to finally derive the effect of physical exercise. Data fusion technology is a hotspot of research and attention in the field of multisensor target tracking, and multisensor target tracking data fusion technology has a broad development prospect. In general, it seems that there are still many problems that need to be studied. In this paper, for the proposed multilevel multisensor information acquisition solution, a three-level information acquisition system from the front-end multisensor to the monitoring station, the surveillance subcenter, and the surveillance center is realized by successively simulating access and accessing multisensor information. Finally, a demonstration and verification environment was built according to the system testing requirements, and the system testing was completed to demonstrate the software operation effect. The experimental results show that the system design is reasonable and feasible, and the software and algorithm research results are effectively usable, which solves the problems of multisensor information acquisition interface diversity and is not easy to expand in multilevel surveillance and achieves the purpose of the research. The research in this paper is only an initial phase of work, and many aspects deserve deeper research and improvement. At the same time, the research work in this paper is only a part of multisensor target tracking data fusion, which needs to be further improved in future research work.

\section{Data Availability}

The data used to support the findings of this study are available from the corresponding author upon request.

\section{Conflicts of Interest}

The authors declare that they have no known competing financial interests or personal relationships that could have appeared to influence the work reported in this paper.

\section{References}

[1] A. Dameshghi and M. H. Refan, "Wind turbine gearbox condition monitoring and fault diagnosis based on multi-sensor information fusion of SCADA and DSER-PSO-WRVM method," International Journal of Modelling and Simulation, vol. 39, no. 1, pp. 48-72, 2019.

[2] N. Xiong and P. Svensson, "Multi-sensor management for information fusion: issues and approaches," Information fusion, vol. 3, no. 2, pp. 163-186, 2002.

[3] Y. Lu, H. Wang, F. Hu, B. Zhou, and H. Xi, "Effective recognition of human lower limb jump locomotion phases based on multi-sensor information fusion and machine learning," Medical \& Biological Engineering \& Computing, vol. 59, no. 4, pp. 883-899, 2021.

[4] S. Sarkar, S. Sarkar, K. Mukherjee, A. Ray, and A. Srivastav, "Multi-sensor information fusion for fault detection in aircraft gas turbine engines," Proceedings of the Institution of Mechanical Engineers, Part G: Journal of Aerospace Engineering, vol. 227, no. 12, pp. 1988-2001, 2013.

[5] K. Jiang, D. Su, and Y. Zheng, "Intelligent acquisition model of traffic congestion information in the vehicle networking environment based on multi-sensor fusion," International Journal of Vehicle Information and Communication Systems, vol. 4, no. 2, pp. 155-169, 2019.

[6] I. V. Maslov and I. Gertner, "Multi-sensor fusion: an evolutionary algorithm approach," Information Fusion, vol. 7, no. 3, pp. 304-330, 2006.

[7] S. McLaughlin, B. Holbert, A. Fawaz, R. Berthier, and S. Zonouz, "A multi-sensor energy theft detection framework for advanced metering infrastructures," IEEE Journal on Selected Areas in Communications, vol. 31, no. 7, pp. 13191330, 2013. 
[8] Y. Zhang, H. Zhang, N. M. Nasrabadi, and T. S. Huang, "Multi-metric learning for multi-sensor fusion based classification," Information Fusion, vol. 14, no. 4, pp. 431-440, 2013.

[9] T. D. Dixon, S. G. Nikolov, J. J. Lewis et al., "Task-based scanpath assessment of multi-sensor video fusion in complex scenarios," Information Fusion, vol. 11, no. 1, pp. 51-65, 2010.

[10] L. R. M. Johansson and N. Xiong, "Perception management: an emerging concept for information fusion," Information Fusion, vol. 4, no. 3, pp. 231-234, 2003.

[11] M. Wei, M. Chen, and D. Zhou, "Multi-sensor information based remaining useful life prediction with anticipated performance," IEEE Transactions on Reliability, vol. 62, no. 1, pp. 183-198, 2013.

[12] E. Nazari, R. Biviji, A. H. Farzin, P. Asgari, and H. Tabesh, "Advantages and challenges of information fusion technique for big data analysis: proposed framework," Journal of Biostatistics and Epidemiology, vol. 7, no. 2, pp. 189-216, 2021.

[13] J. A. Rodger, "Toward reducing failure risk in an integrated vehicle health maintenance system: a fuzzy multi-sensor data fusion Kalman filter approach for IVHMS," Expert Systems with Applications, vol. 39, no. 10, pp. 9821-9836, 2012.

[14] L. Gao, A. K. Bourke, and J. Nelson, "Evaluation of accelerometer based multi-sensor versus single-sensor activity recognition systems," Medical Engineering \& Physics, vol. 36, no. 6, pp. 779-785, 2014.

[15] J. J. Guiry, P. Van de Ven, and J. Nelson, "Multi-sensor fusion for enhanced contextual awareness of everyday activities with ubiquitous devices," Sensors, vol. 14, no. 3, pp. 5687-5701, 2014.

[16] S. Roy, D. Sarkar, and D. De, "Entropy-aware ambient IoT analytics on humanized music information fusion," Journal of Ambient Intelligence and Humanized Computing, vol. 11, no. 1, pp. 151-171, 2020.

[17] O. Banos, M. Damas, A. Guillen et al., "Multi-sensor fusion based on asymmetric decision weighting for robust activity recognition," Neural Processing Letters, vol. 42, no. 1, pp. 5$26,2015$.

[18] J. Triloka, S. M. N. A. Senanayake, and D. Lai, "Neural computing for walking gait pattern identification based on multisensor data fusion of lower limb muscles," Neural Computing and Applications, vol. 28, no. S1, pp. 65-77, 2017.

[19] G. Fasano, D. Accardo, A. Moccia et al., "Multi-sensor-based fully autonomous non-cooperative collision avoidance system for unmanned air vehicles," Journal of Aerospace Computing, Information, and Communication, vol. 5, no. 10, pp. 338360, 2008.

[20] F. Miao, Z. D. Liu, J. K. Liu, B. Wen, Q. Y. He, and Y. Li, "Multi-sensor fusion approach for cuff-less blood pressure measurement," IEEE Journal of Biomedical and Health Informatics, vol. 24, no. 1, pp. 79-91, 2020.

[21] A. Belmonte-Hernandez, G. Hernandez-Penaloza, F. Alvarez, and G. Conti, "Adaptive fingerprinting in multi-sensor fusion for accurate indoor tracking," IEEE Sensors Journal, vol. 17, no. 15, pp. 4983-4998, 2017. 